Review Article

\title{
Drug-Coated Balloon Angioplasty and Debulking for the Treatment of Femoropopliteal In-Stent Restenosis: A Systematic Review and Meta-Analysis
}

\author{
Zhu Tong, Lianrui Guo, Lixing Qi, Shijun Cui, Xixiang Gao, Yang Li, Jianming Guo, \\ and Yongquan $\mathrm{Gu}$ (D)
}

Department of Vascular Surgery, Xuanwu Hospital, Capital Medical University, Beijing 100053, China

Correspondence should be addressed to Yongquan Gu; guyongquan@xwhosp.org

Received 25 June 2019; Revised 31 March 2020; Accepted 18 April 2020; Published 10 June 2020

Academic Editor: Giovanni Mariscalco

Copyright @ 2020 Zhu Tong et al. This is an open access article distributed under the Creative Commons Attribution License, which permits unrestricted use, distribution, and reproduction in any medium, provided the original work is properly cited.

\begin{abstract}
The purpose of this article was to compare the efficiency and safety of drug-coated balloon angioplasty (DCB) and atherectomy with percutaneous transluminal angioplasty (PTA) in patients with femoropopliteal in-stent restenosis (ISR). Pubmed, Embase, and the Cochrane Central Register of Controlled Trials (CENTRAL) (all up to March 2019) were searched systematically. Trial sequential analysis (TSA) was conducted. 5 studies with 599 participants were included. Compared with PTA, DCB significantly increased the rate of patency (6 months: RR 1.65, 95\% CI 1.30 to 2.09, $P<0.01 ; 12$ months: RR 2.38, 95\% CI 1.71 to $3.30, P<0.01$ ) and the rate freedom from target lesion revascularization (TLR) (6 months: RR 1.18, 95\% CI 1.09 to $1.28, P<0.01 ; 12$ months: RR 1.56, $95 \%$ CI 1.33 to $1.82, P<0.01)$ at 6 and 12 months follow-up, and the TSA results showed these outcomes were reliable. The rate of clinical improvement by $\geq 1$ Rutherford category in the DCB group was higher than that in the PTA group (6 months: RR 1.35, 95\% CI 1.03 to $1.75, P=0.03 ; 12$ months: RR $1.46,95 \%$ CI 1.17 to $1.82, P<0.01$ ) at 6 and 12 months. There is no statistically difference of ABI, all-cause mortality, and incidence of amputation between DCB group and PTA group (MD $0.03,95 \%$ CI - 0.03 to $0.08, P=0.40$; RR $1.24,95 \%$ CI 0.46 to $3.34, P=0.67$; RR $0.32,95 \%$ CI 0.01 to $7.61, P=0.48$ ). Compared with PTA, the rate of patency and freedom from TLR in the laser atherectomy (LD) group was higher than that in the PTA group (patency: 6 months: RR 1.28, 95\% CI 1.01 to $1.64, P<0.05,12$ months: RR 2.25, 95\% CI 1.14 to 4.44, $P<0.05$; freedom from TLR: 6 months: RR 1.27 , 95\% CI 1.05 to $1.53, P=0.01,12$ months: RR $1.59,95 \%$ CI 1.12 to $2.25, P=0.01$ ) at 6 and 12 months follow-up. In conclusion, DCB and LD had superior clinical (freedom from TLR and clinical improvement) and angiographic outcomes (patency rate) compared with PTA for the treatment of femoropopliteal ISR. Moreover, DCB and LD had a low incidence of amputation and mortality and were relatively safe methods.
\end{abstract}

\section{Introduction}

Self-expanding nitinol stent is increasingly used for the treatment of symptomatic femoropopliteal arterial occlusive disease because of its reduction of stent fracture and other procedural complications of earlier devices. Despite these clear benefits, in-stent restenosis (ISR) remains a challenging clinical problem. Worldwide, more than 400000 stents are implanted in femoropopliteal arterial annually, 30\%-40\% of which will develop ISR within 2-3 years of implantation [1].

Different from de novo lesions closely associated with atherosclerosis, ISR lesions are predominantly caused by neointimal hyperplasia, which consists primarily of smooth muscle cells and hydrated collagen matrix [2]. And ISR lesions are usually long and highly calcified. Conventional endovascular therapies, such as percutaneous transluminal angioplasty (PTA) and repeat stenting, as methods of treating femoropopliteal ISR have no satisfactory clinical outcomes. Several prospective single-arm $[3,4]$ and retrospective trials $[5,6]$ have introduced methods of drug-coated balloon angioplasty (DCB, carrying antiproliferative drug, usually paclitaxel), or atherectomy (directional or laser) for the treatment of femoropopliteal ISR and had promising results. In recent years, some prospective controlled trials compared the 
effectiveness and safety of DCB, and atherectomy with PTA in the treatment of femoropopliteal ISR. However, the results are inconclusive [7-9].

In consideration of these inconclusive results, we carried out this meta-analysis of the prospective controlled trials including patients with femoropopliteal ISR treated with either DCB and atherectomy or PTA.

\section{Materials and Methods}

2.1. Protocol and Registration. Our meta-analysis was conducted according to the Preferred Reporting Items for Systematic reviews and meta-analyses (PRISMA) recommendations. A protocol for this meta-analysis has been registered on PROSPERO (http://www.crd.york.ac.uk/prospero) and the registration number is CRD42019128171.

2.2. Search Strategy. Pubmed, Embase, and the Cochrane Central Register of Controlled Trials (CENTRAL) (all up to March 2019) were searched according to the guidelines of the Cochrane Handbook, without language restrictions. The following subject headings and keywords: "restenosis" were used, "superficial femoral artery", "popliteal artery", "femoropopliteal artery" et al. A supplementary search of the reference lists from all retrieved trials and reviews was also performed. In case the articles were not available from databases, we directly contacted the corresponding authors by mail. All results were imported into Endnote X9 (Thomson Reuters, New York, USA) for the exclusion of duplicates, and subsequently, we screened the titles, abstracts, and fulltexts of eligible trials.

2.3. Inclusion and Exclusion Criteria. Inclusion criteria were (1) prospective controlled studies; (2) patients with femoropopliteal ISR; (3) treatment methods of experiments were DCB or atherectomy; (4) a minimum follow-up of 6 months.

2.4. Endpoints and Data Extraction. The primary endpoints were patency at 6 and 12 months, and freedom from target lesion revascularization (TLR) at 6 and 12 months. The secondary endpoints included clinical improvement by $\geq 1$ Rutherford category at 6 and 12 months, ankle-brachial index $(\mathrm{ABI})$ at 6 and 12 months, all-cause mortality at 6 and 12 months, and amputation. Data including date of publication, name of the first author, study type, patient characteristics (mean age, number of patients and sex ratio, basic diseases), inclusion criteria, exclusion criteria, and characteristics of ISR lesions. And the above endpoints were extracted from the eligible studies using a standard data extraction form.

Database search, eligibility evaluation, and data extraction were performed independently by two authors (database search was conducted by Zhu Tong and Lianrui Guo; eligibility evaluation was conducted by Xixiang Gao and Zhu Tong; data extraction was conducted by Lixing Qi, Shijun Cui); lack of consensus was resolved by a third author.

2.5. Data Synthesis and Statistical Analysis. Risk ratio (RR) with $95 \%$ confidence intervals (CIs) and standardized mean difference (SMD) with 95\% CIs were used for the expression of dichotomous and continuous variables, respectively. Get- data software was used to get data on condition that data were presented as statistical graph only and were not procured from the corresponding author. A $P$ value $<0.05$ was considered as statistically significant. The heterogeneity among trials was assessed with the Cochran's Q-statistic test and the $I 2$ test. If $I 2$ was more than $50 \%$ or $P$ value ( $Q$-test) was less than 0.05 , we thought high heterogeneity existed and a random-effects model was adopted. Otherwise, a fixed effects model was used. Subgroup analysis was performed according to the treatment method (DCB or atherectomy) of experiment group to establish the derivation of heterogeneity. The statistical analysis was conducted using RevMan software (version 5.1; Cochrane Collaboration, Copenhagen, Denmark). Trial sequential analysis (TSA) was conducted to control the risks of type I and type II errors and calculate required information size (RIS) [10].

2.6. Risk of Bias. Two authors independently assessed the following seven categories of risk of bias according to the Cochrane guidelines, and lack of consensus was resolved in group discussions. The risk of bias was classified in the following seven categories: (1) random sequence generation, (2) allocation concealment, (3) blinding of participants and personnel, (4) blinding of outcome assessment, (5) incomplete outcome data, (6) selective outcome reporting, (7) other sources of bias. Each category can be graded into three levels: low risk, unclear risk, or high risk.

\section{Results}

3.1. Study Selection. 2033 articles were identified initially. Finally, a total of 5 studies with 599 participants were included in this meta-analysis [7-9, 11, 12]. The detailed process of literature search is summarized in Figure 1.

3.2. Study Characteristics. 4 studies compared the treatment method of DCB and PTA, 1 compared debulking and PTA. Patients included these studies were elderly, and most of them had disease of dyslipidemia and hypertension. The clinical and angiographic characteristics of patients between groups in each study were comparable. More details are summarized in Table 1.

3.3. Risk of Bias and Quality of Evidence. The risk of bias of included studies was showed in Figure 2.

\subsection{Clinical Outcomes}

3.4.1. Patency. Four studies reported vessel patency at 6 months follow-up. The rate of patency in the DCB group was significantly higher than that in the PTA group (RR $1.65,95 \%$ CI 1.30 to $2.09, P<0.01)$ using a fixed effects model $(I 2=0 \%, P=0.78)$ (Figure $3(\mathrm{a}))$. TSA was conducted and the RIS was 542 participants. The cumulative $Z$ curve (blue line) crossed the trial sequential monitoring boundaries (red inward slash) before the RIS has been reached (Figure 3(b)), indicating that positive result has been achieved in advance and further randomized controlled trials (RCTs) are unnecessary. The rate of patency in the debulking 


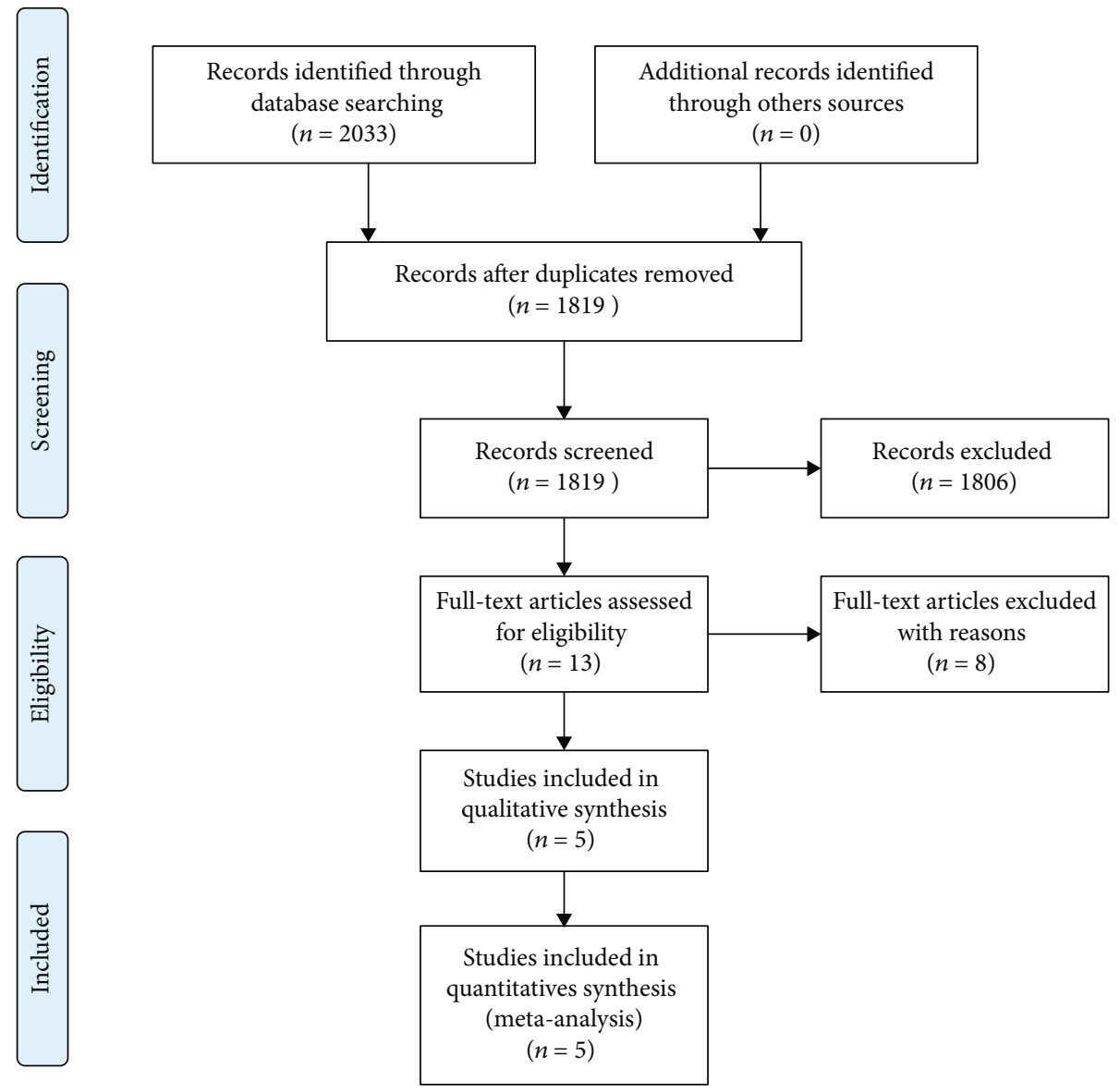

FIgURE 1: PRISMA flow chart.

group was higher than that in the PTA group (RR 1.28, 95\% CI 1.01 to $1.64, P<0.05$ ) (Figure $3(\mathrm{a})$ ).

Four studies reported vessel patency at 12 months followup. The rate of patency in the DCB group was significantly higher than that in the PTA group (RR 2.38, 95\% CI 1.71 to $3.30, P<0.01)$ using a fixed effects model $(I 2=0 \%, P=0.41)$ (Figure 4(a)). TSA was conducted and the RIS was 1144 participants. The result of TSA indicating that positive result has been achieved in advance and further RCTs are unnecessary (Figure 4(b)). The rate of patency in the debulking group was higher than that in the PTA group (RR 2.25, 95\% CI 1.14 to $4.44, P<0.05$ ) (Figure $4(\mathrm{a})$ ).

3.5. Freedom from TLR. Five studies reported freedom from TLR at 6 months follow-up. The rate of freedom from TLR in the DCB group was significantly higher than that in the PTA group (RR 1.18, 95\% CI 1.09 to $1.28, P<0.01)$ using a fixed effects model $(I 2=0 \%, P=0.71)$ (Figure 5(a)). TSA was conducted and the RIS was 287 participants. The cumulative $\mathrm{Z}$ curve (blue line) crossed the trial sequential monitoring boundaries (red inward slash) and the RIS has been reached (Figure 5(b)). Consequently, we could think that our result is reliable and no more RCTs are needed. The rate of freedom from TLR in the debulking group was higher than that in the PTA group (RR 1.27, 95\% CI 1.05 to $1.53, P=0.01$ ) (Figure 5(a)).
Five studies reported freedom from TLR at 12 months follow-up. The rate of freedom from TLR in the DCB group was significantly higher than that in the PTA group (RR 1.56, 95\% CI 1.33 to $1.82, P<0.01)$ using a fixed effects model $(I 2=45 \%, P=0.14$ ) (Figure 6(a)). TSA was conducted and the RIS was 640 participants. The result of TSA indicating that positive result has been achieved in advance, and further RCTs are unnecessary (Figure 6(b)). The rate of freedom from TLR in the debulking group was higher than that in the PTA group (RR $1.59,95 \%$ CI 1.12 to $2.25, P=0.01$ ) (Figure 6(a)).

3.6. ABI. Three studies reported ABI at 6 months and two studies reported ABI at 12 months. There is no statistical difference of ABI between DCB group and PTA group (MD $0.03,95 \% \mathrm{CI}-0.03$ to $0.08, P=0.40)$ using a fixed effects model $(I 2=0 \%, P=0.44)$ at 6 months (Figure 7$)$ and (MD $-0.04,95 \%$ CI -0.11 to $0.03, P=0.22)$ using a fixed effects model $(I 2=0 \%, P=0.89)$ at 12 months (Figure 8$)$.

There is no statistical difference of ABI between the debulking group and PTA group (MD -0.06, 95\% CI -0.14 to $0.02, P=0.14$ ) at 6 months (Figure 7 ).

3.7. Clinical Improvement by $\geq 1$ Rutherford Category. Two studies reported clinical improvement by $\geq 1$ Rutherford category at 6 months, and three studies reported clinical improvement by $\geq 1$ Rutherford category at 12 months. The 

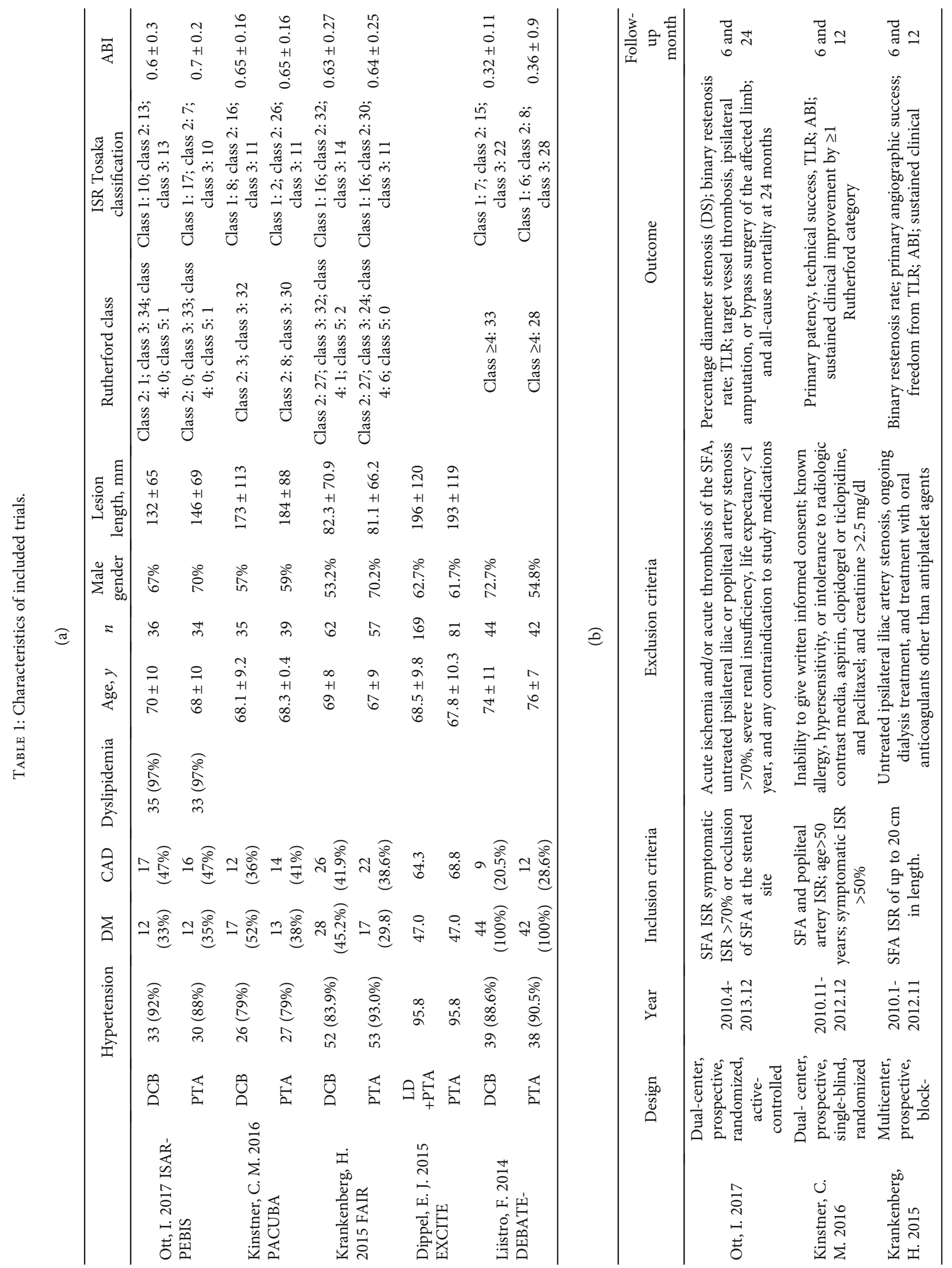


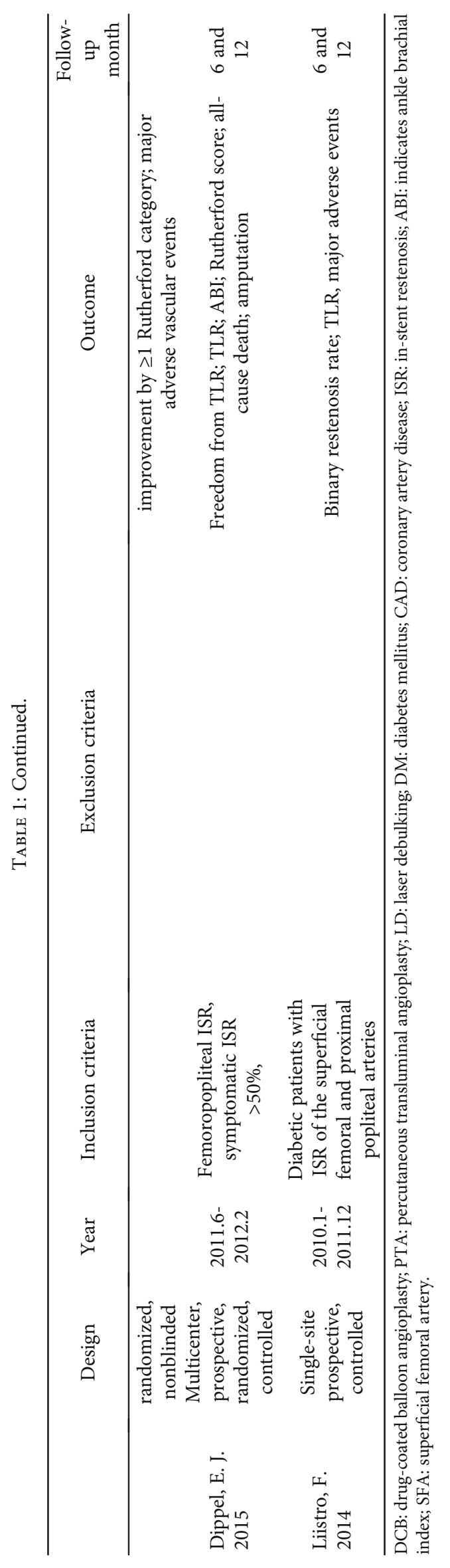




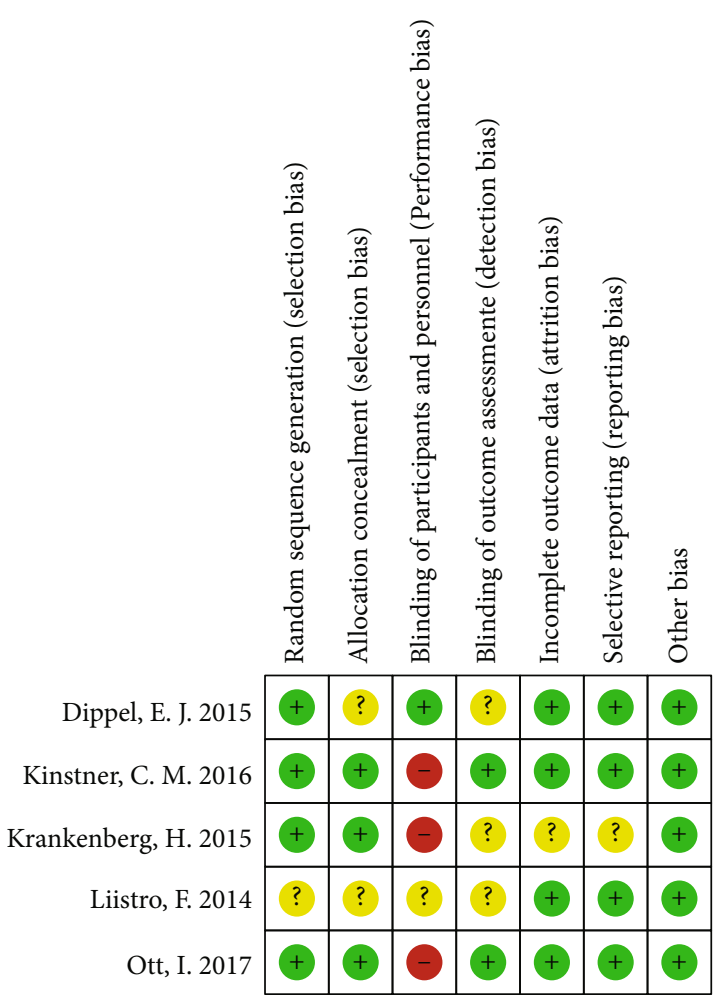

FIGURE 2: Risk of bias summary.

rate of clinical improvement by $\geq 1$ Rutherford category in the DCB group was significantly higher than that in the PTA group (RR $1.35,95 \%$ CI 1.03 to $1.75, P=0.03$ and RR $1.46,95 \% \mathrm{CI} 1.17$ to $1.82, P<0.01)$ using a fixed effects model $(I 2=0 \%, P=0.38$ and $I 2=0 \%, P=0.56)$ at 6 and 12 months (Figures 9 and 10).

\section{Adverse Events}

4.1. All-Cause Mortality. Four studies reported all-cause mortality. There was no statistical difference of all-cause mortality between DCB group and PTA group (RR 1.24, 95\% CI 0.46 to $3.34, P=0.67)$ using a fixed effects model $(I 2=0 \%, P=0.37)$ (Figure 11).

4.2. Amputation. Four studies reported the incidence of amputation. There was no statistically difference of incidence of amputation between DCB group and PTA group (RR 0.32, 95\% CI 0.01 to $7.61, P=0.48)$ and between debulking group and PTA group (RR 0.17, 95\% CI 0.02 to $1.35, P=0.09$ ) using a fixed effects model (Figure 12).

4.3. Publication Bias. Egger's funnel plot showed no publication bias (Additional file).

\section{Discussion}

In the present meta-analysis, we evaluated the efficacy and safety of two new therapies (DCB and debulking) versus PTA in patients suffering from femoropopliteal ISR. DCB, firstly used in coronary arteries, delivers antiproliferative drug, usually paclitaxel at the site of lesions, which could inhibit smooth muscle cell replication and proliferation and therefore reduce the incidence of restenosis [13]. In the following years, researchers found the superiority of DCB for the treatment of coronary ISR to PTA $[14,15]$. Because of the ability of locally drug delivery and promising results in the treatment of coronary ISR, DCB had attracted attention and was tentatively used in the treatment of femoropopliteal ISR.

Four studies comparing DCB with PTA were included in this meta-analysis. The key findings are that in patients with femoropopliteal ISR. (1) In comparison to PTA, DCB had a higher patency rate at 6 and 12 months follow-up, and TSA results showed that our outcomes were reliable and no more RCTs are required. (2) DCB increased the rate of freedom from TLR significantly compared with PTA at 6 and 12 months follow-up, and TSA results showed that our outcomes were reliable and no more RCTs are required. (3) Clinical improvement in the DCB group was superior to that in the PTA group. (4) The ABI in two groups was both below normal (but above baseline), and no difference was found between two groups at 6 and 12 months follow-up, indicating that DCB did not significantly increase the ABI and improve Ischemic condition, which seemed contradictory to clinical outcomes (freedom from TLR and clinical improvement) and angiographic outcomes (patency rate). (5) There was no difference in mortality, incidence of amputation, and incidence of all-cause adverse events between two groups.

Debulking therapy includes directional atherectomy (DA) and laser atherectomy (LD). Directional atherectomy works through cutting blades which resects the obstructive plaque longitudinally [16]. Different from directional atherectomy, laser atherectomy works through laser-guided photoablation which breaks down and vaporizes the obstructive plaque [17]. Debulking therapy has unique advantages used in the treatment of ISR such as significant removing of hyperplastic neointima, which is the predominant composition of ISR lesions, enlarging the lumen volume and recanalizing the vessel. To now, EXCITE-ISR is the unique prospective controlled study comparing the efficacy of debulking and PTA. The result showed that at 6 months compared with PTA, the method of LD had higher rate of patency $(71.1 \%$ vs $56.4 \%, P=0.004)$ and lower rate of TLR $(20.2 \%$ vs $36.3 \%$, $P=0.003)$. However, the same as DCB, LD did also not significantly increase the $\mathrm{ABI}$ and improve Ischemic condition.

Our findings are in accord with the results of the study of Shahab Hajibandeh et al. which has a conclusion that compared with PTA, DCB and LD both confer improved outcomes [18]. Although DCB has a distinct superiority, Charles Nicolais et al. thought it is not the first choice for the treatment of ISR. Their findings suggested that repeat stenting with second-generation drug-eluting stents (DES) is more likely to have optimal outcomes. DCB might only be an effective alternative on condition that repeat stenting is not preferable [19]. However, to now, there are no trials to compare the efficiency and safety of DCB and repeat stenting with DES. So more high-quality RCTs are needed to evaluate these two methods.

According to the findings of this meta-analysis, we have some questions. 


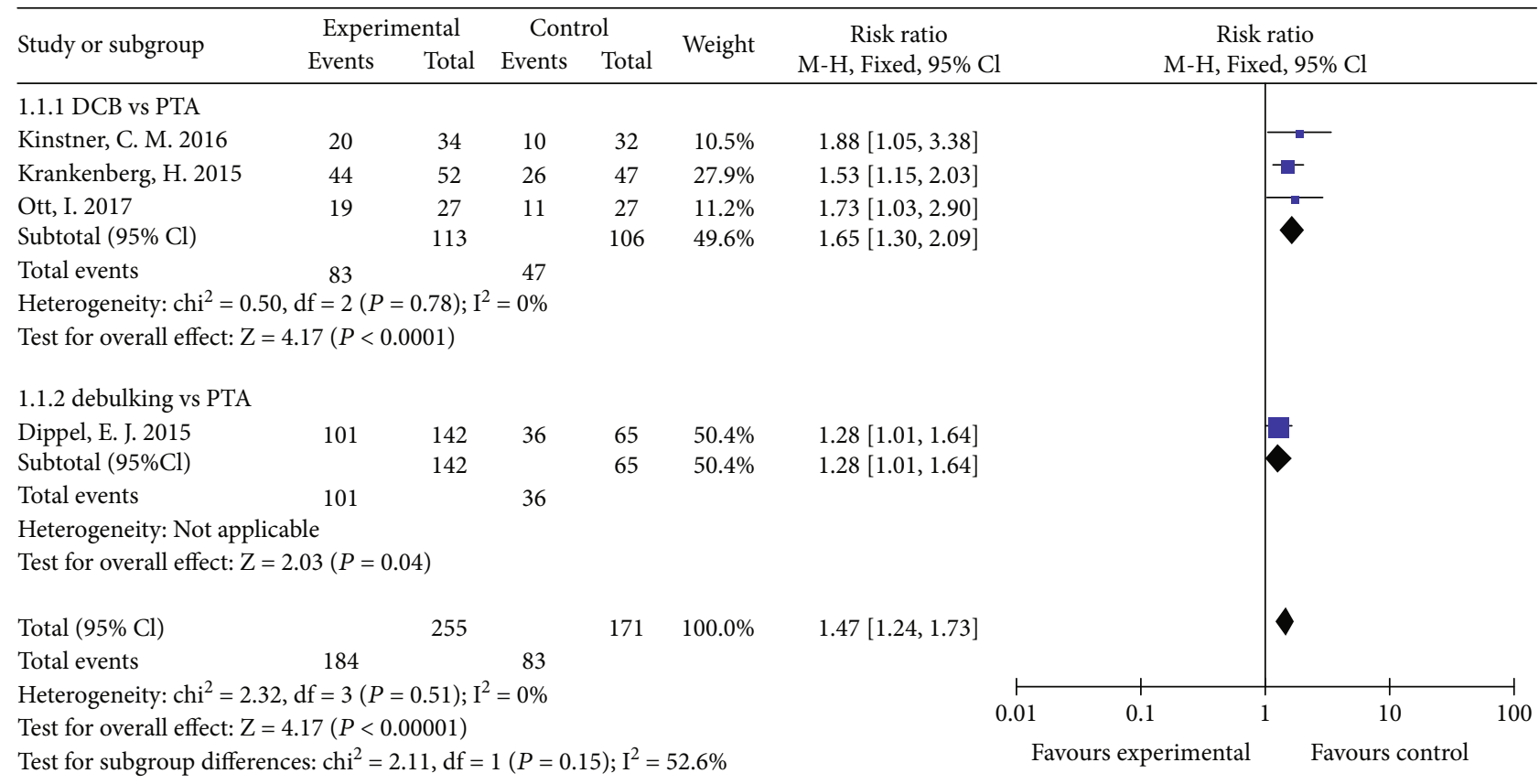

(a)

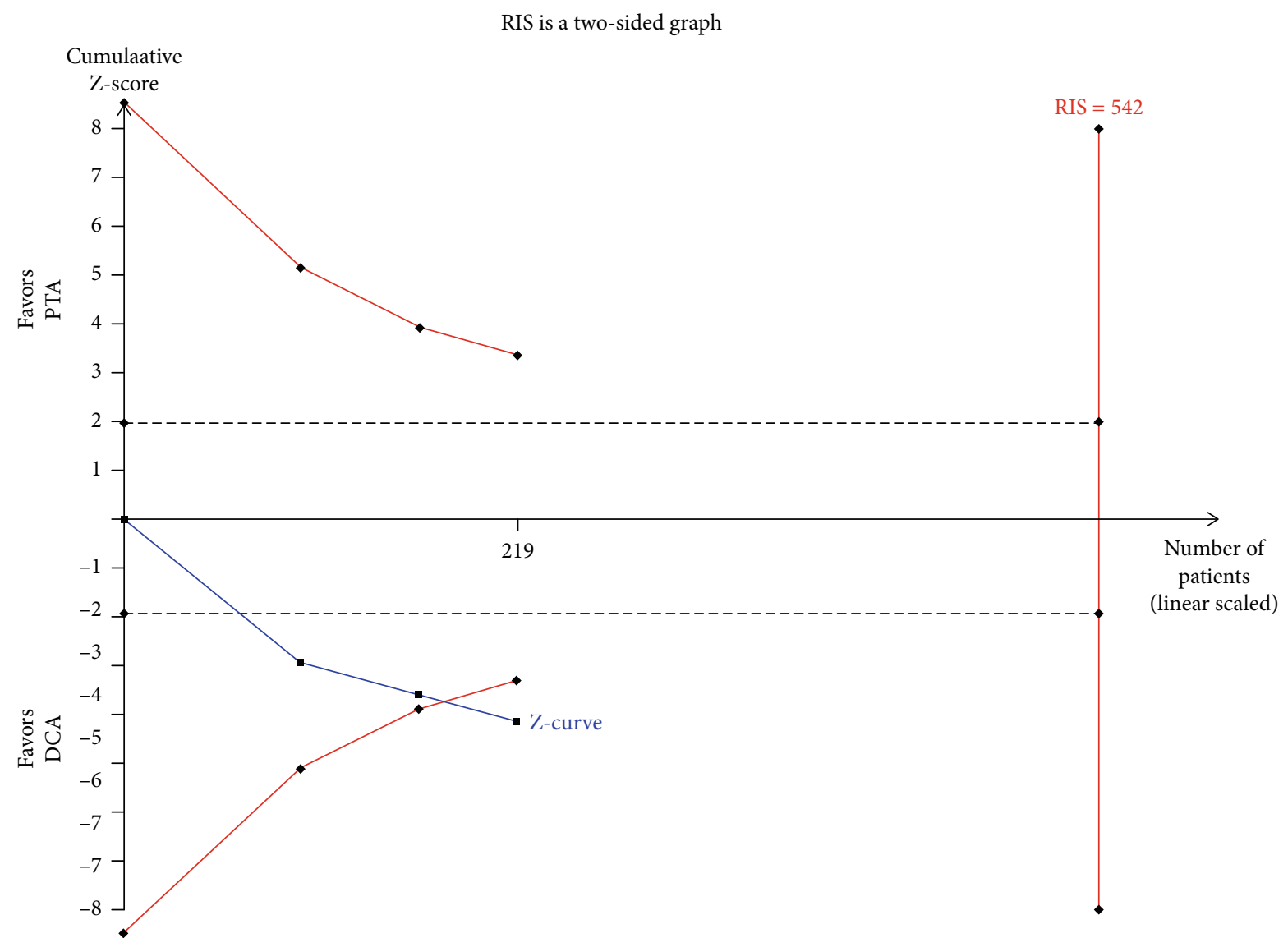

(b)

Figure 3: (a) Forest plot for patency at 6 months. M-H: Mantel-Haenszel test; Fixed: a fixed effects model; CI: confidence intervals. (b) TSA for patency at 6 months. TSA: trial sequential analysis. 


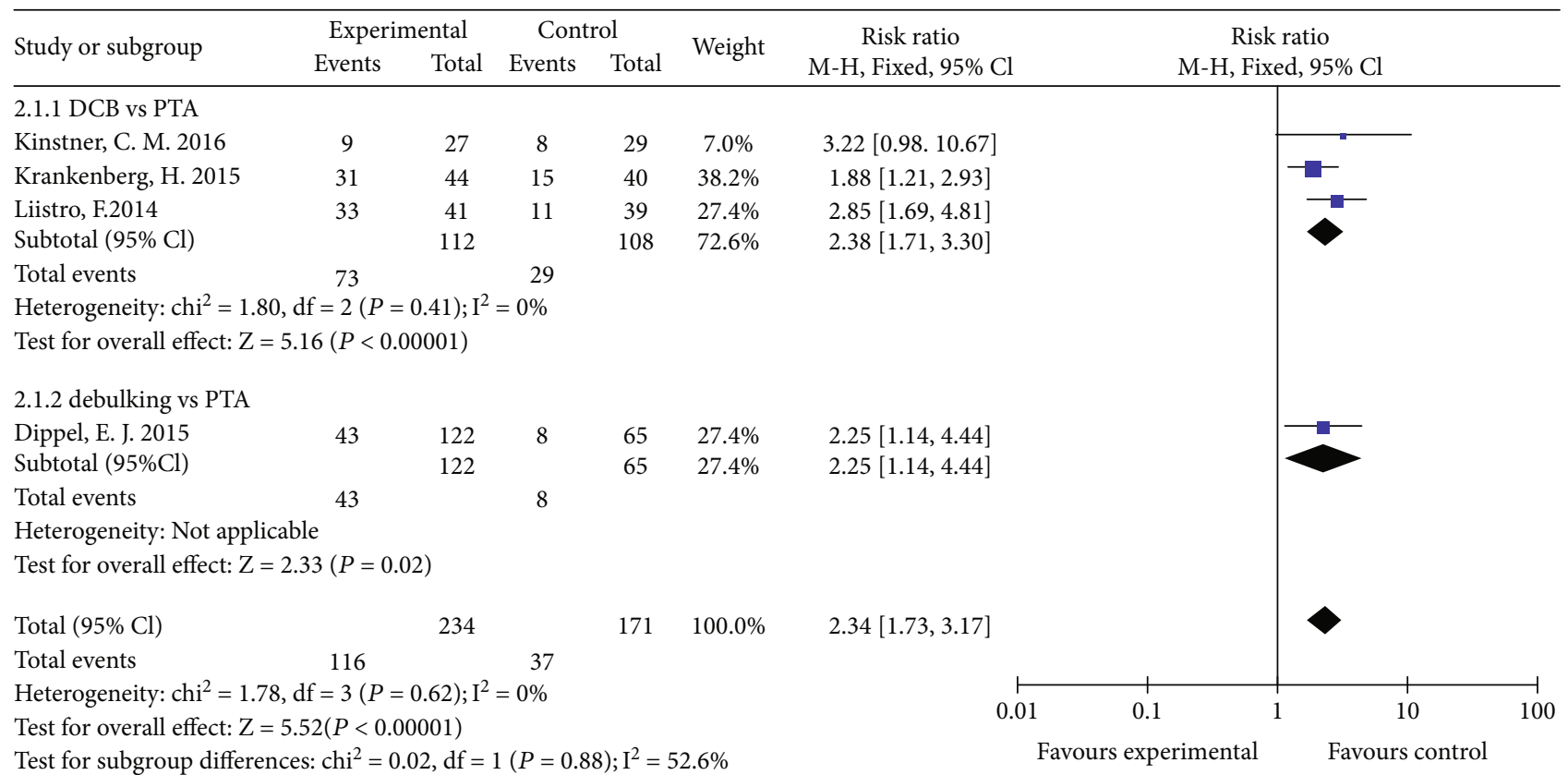

(a)

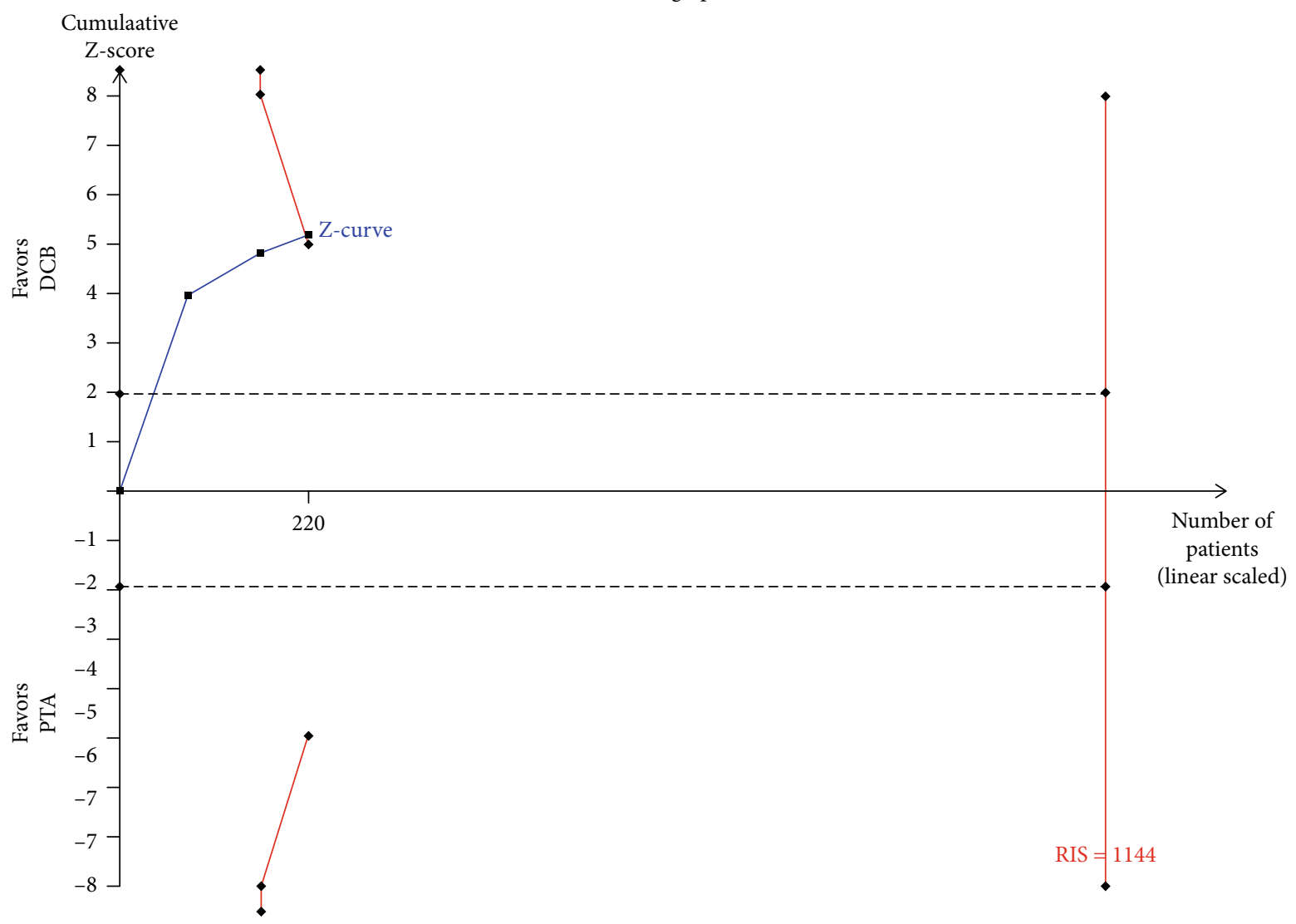

(b)

Figure 4: (a) Forest plot for patency at 12 months. M-H: Mantel-Haenszel test; Fixed: a fixed effects model; CI: confidence intervals. (b) TSA for patency at 6 months. TSA: trial sequential analysis.

First, although DCB and LD could significantly increase the rate of patency and freedom from TLR, their shortcomings are also not negligible. Firstly, DCB is still a method of balloon angioplasty and for the occupation of substantial hyperplastic tissues, its antiproliferative effect cannot be effectively exerted. Next, DCB alone has certain of procedural 


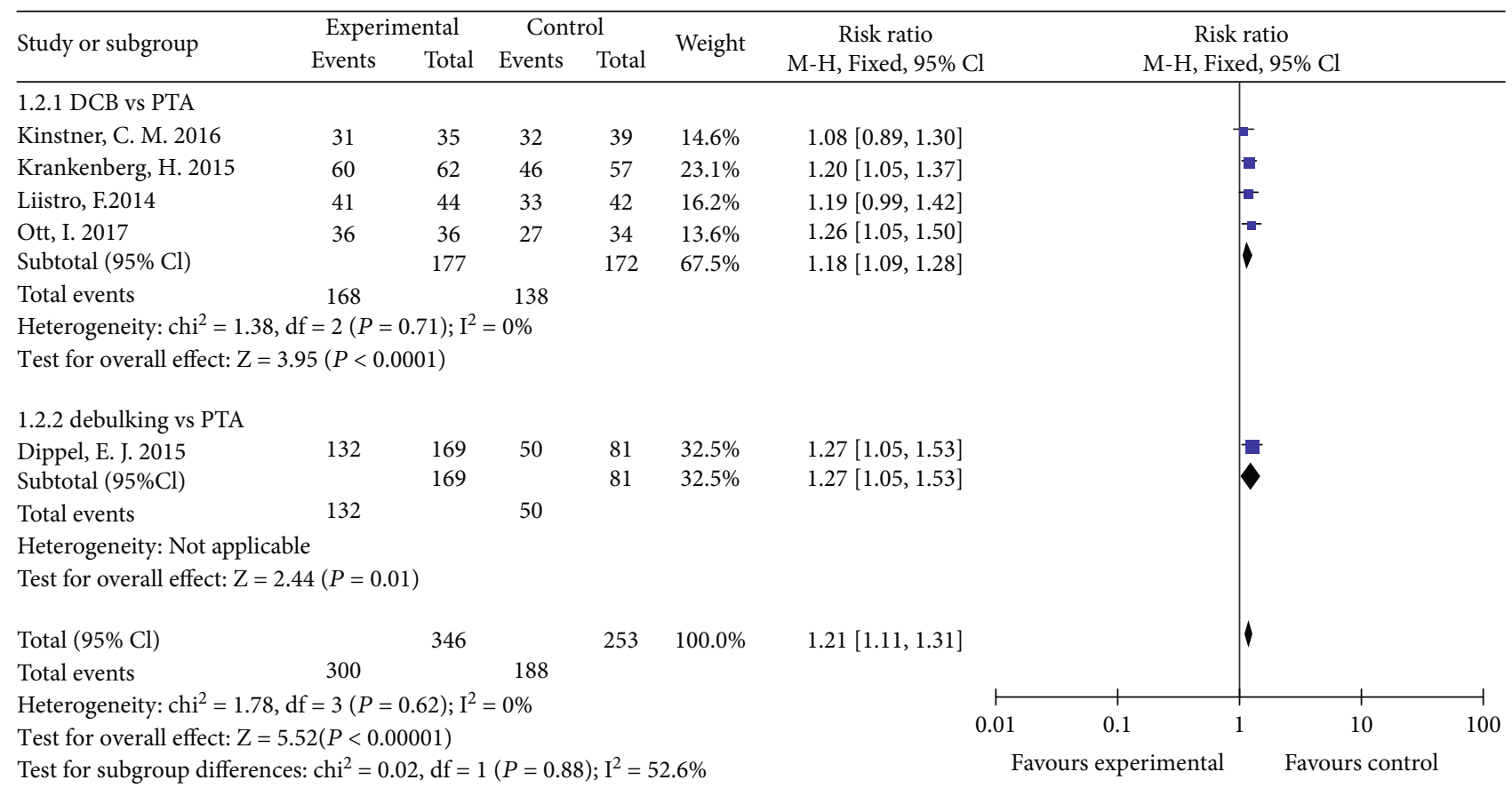

(a)

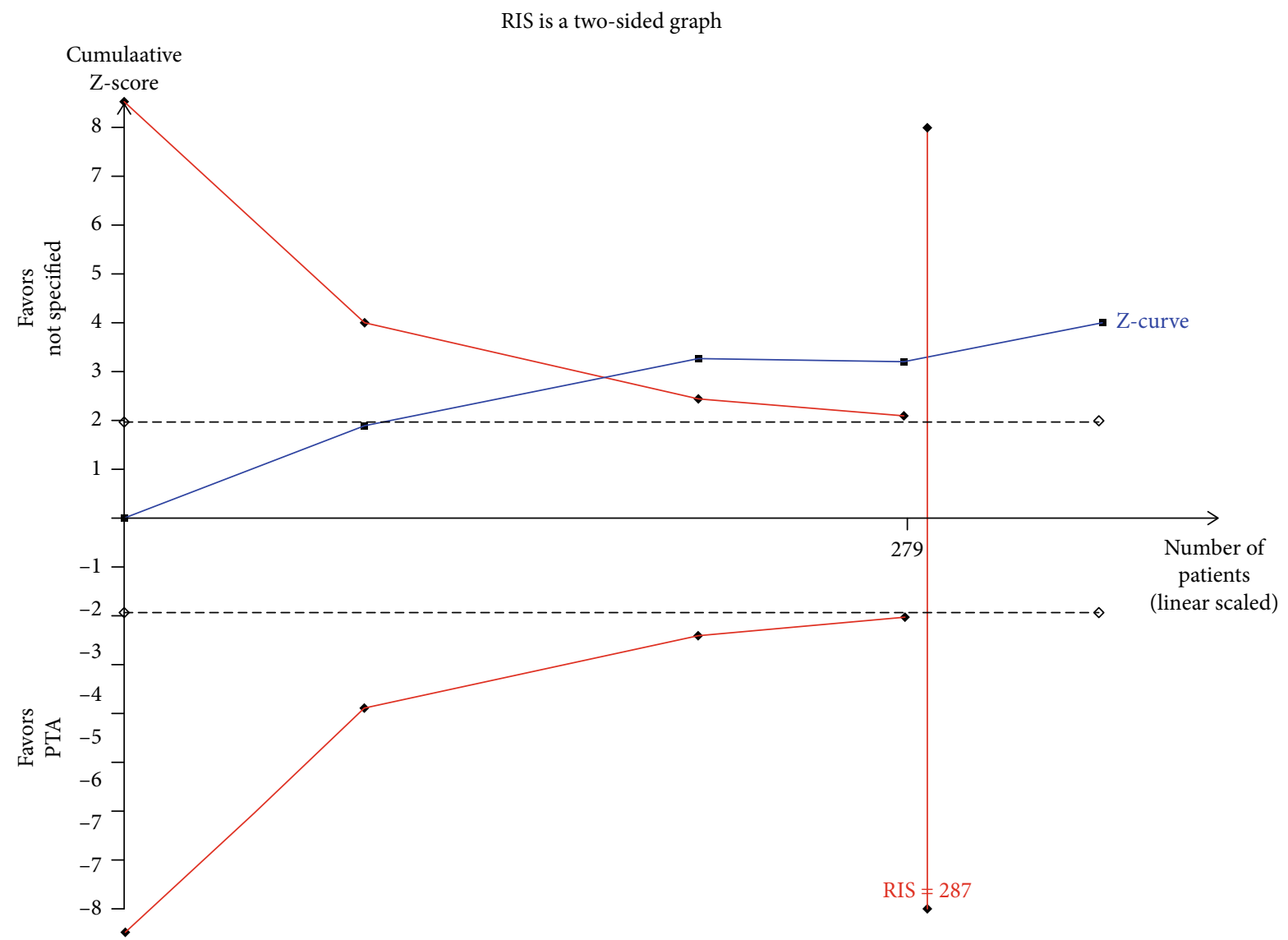

(b)

Figure 5: (a) Forest plot for freedom from TLR at 6 months. M-H: Mantel-Haenszel test; Fixed: a fixed effects model; CI: confidence intervals; TLR: target lesion revascularization. (b) TSA for freedom from TLR at 6 months. TSA: trial sequential analysis; TLR: target lesion revascularization. 


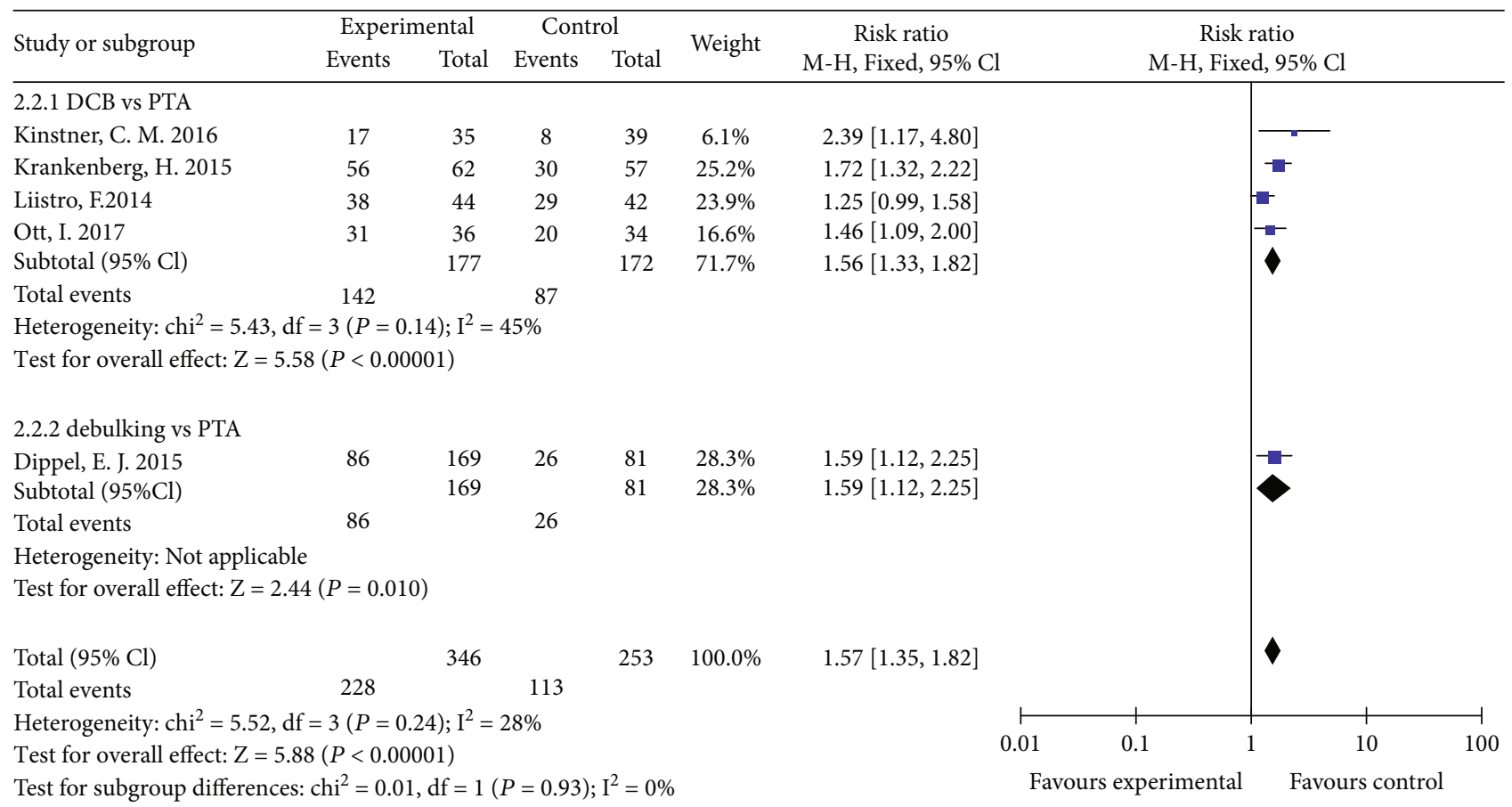

(a)

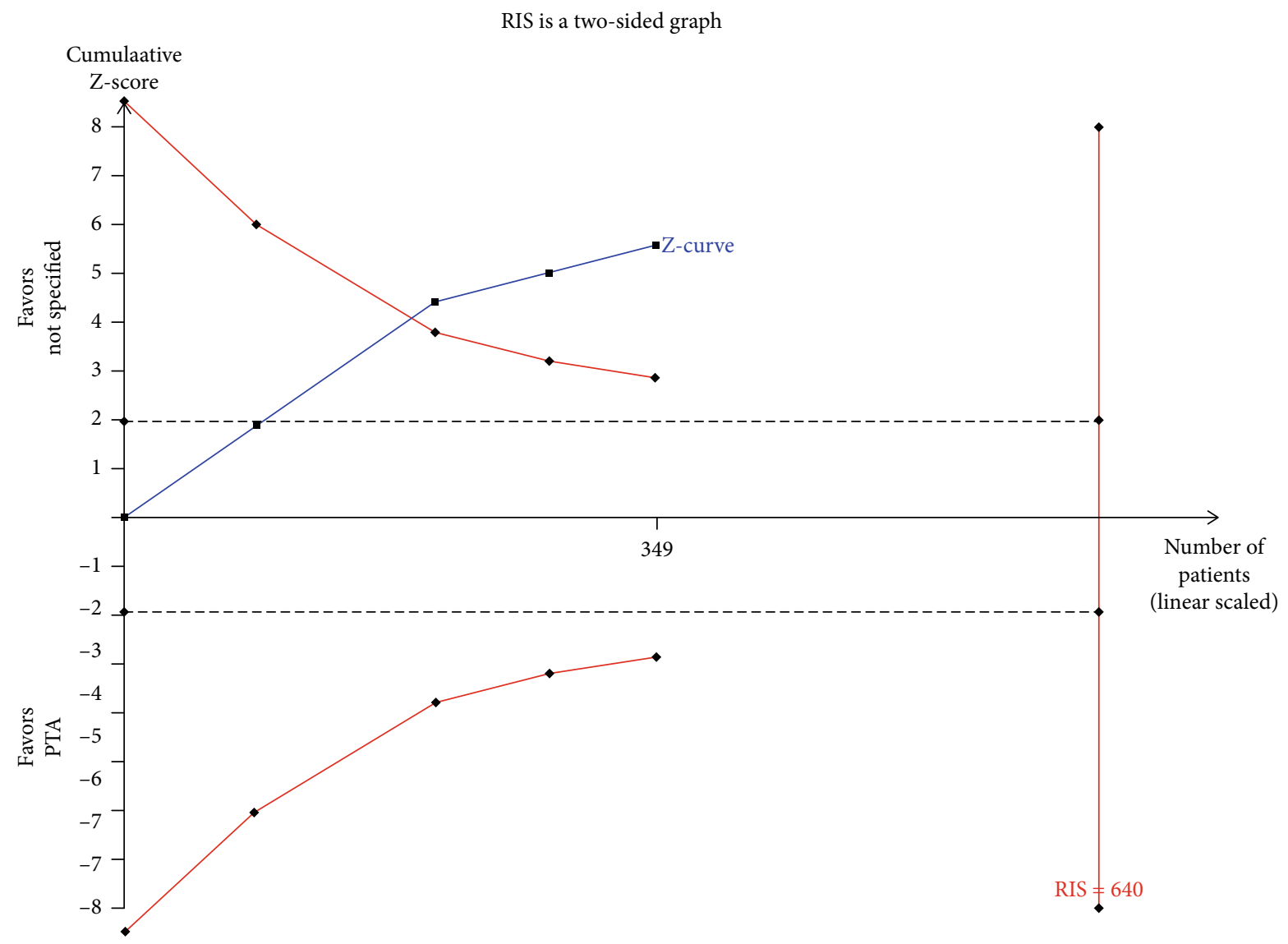

(b)

Figure 6: (a) Forest plot for freedom from TLR at 12 months. M-H: Mantel-Haenszel test, Fixed: a fixed effects model, CI: confidence intervals, TLR: target lesion revascularization. (b) TSA for freedom from TLR at 12 month. TSA: trial sequential analysis, TLR: target lesion revascularization. 


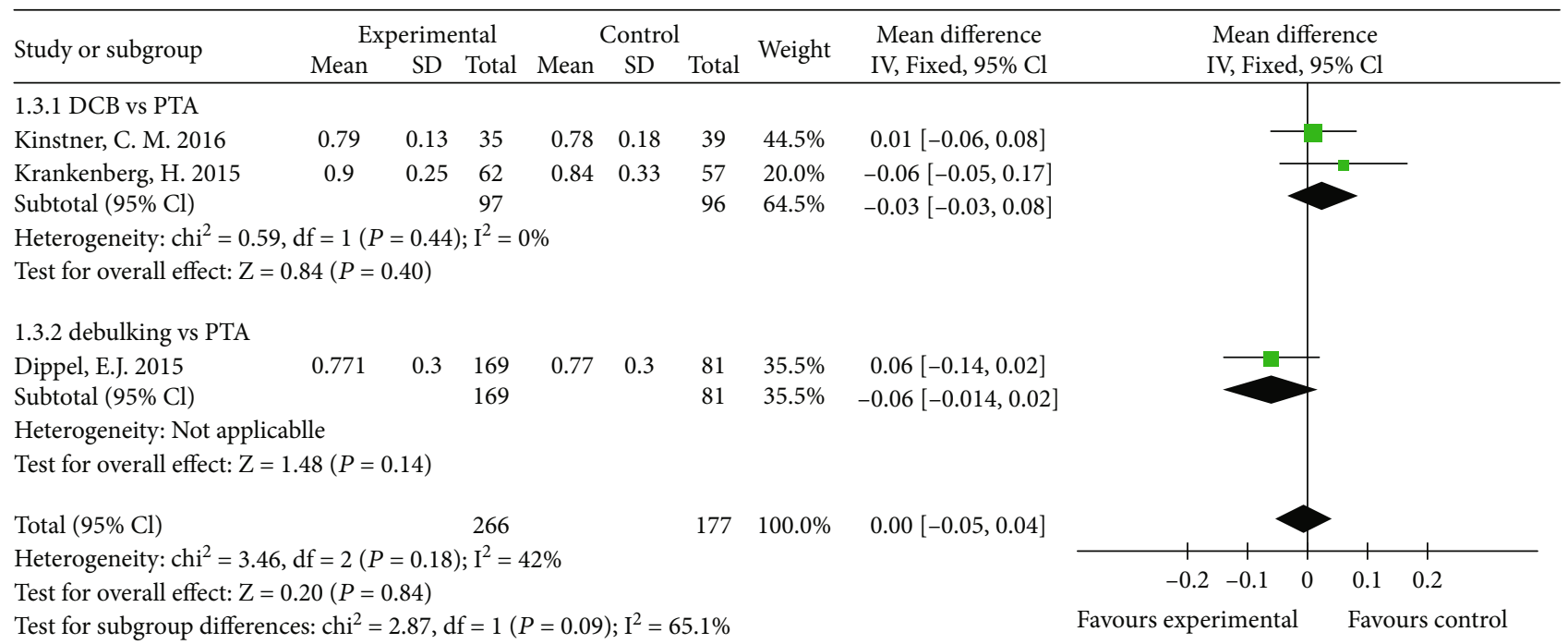

FIGURE 7: Forest plot for ABI at 6 month. IV: inverse variance; Fixed: a fixed effects model; CI: confidence intervals; ABI: ankle-brachial index.

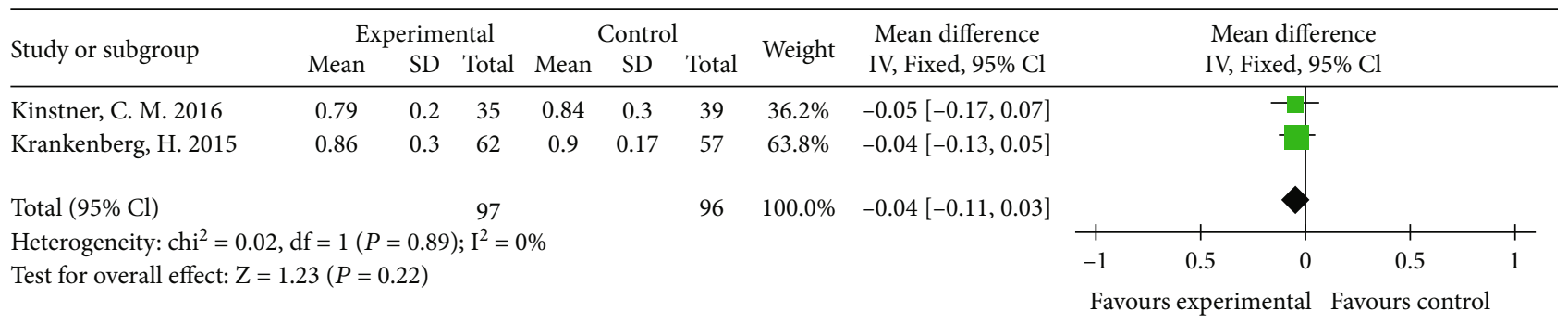

FIgURE 8: Forest plot for ABI at 12 month. IV: inverse variance; Fixed: a fixed effects model; CI: confidence intervals, ABI: ankle-brachial index.

\begin{tabular}{|c|c|c|c|c|c|c|c|c|c|c|}
\hline \multirow{2}{*}{ Study or subgroup } & \multicolumn{2}{|c|}{ Experimental } & \multicolumn{2}{|c|}{ Control } & \multirow{2}{*}{ Weight } & \multirow{2}{*}{$\begin{array}{c}\text { Risk ratio } \\
\text { M-H, Fixed, 95\% Cl }\end{array}$} & \multirow{2}{*}{\multicolumn{3}{|c|}{$\begin{array}{c}\text { Risk ratio } \\
\text { M-H, Fixed, 95\% Cl }\end{array}$}} & \\
\hline & Events & Total & Events & Total & & & & & & \\
\hline Kinstner, C. M. 2016 & 20 & 35 & 14 & 39 & $32 \%$ & $1.59[0.96,2.65]$ & & & & \\
\hline Krankenberg, H. 2015 & 36 & 51 & 27 & 47 & $68 \%$ & $1.23[0.91,1.66]$ & & & & \\
\hline Total $(95 \% \mathrm{Cl})$ & & 86 & & 86 & $100.0 \%$ & $1.35[1.03,1.75]$ & & & & \\
\hline Total events & 56 & & 41 & & & & & & & \\
\hline
\end{tabular}

FIGURE 9: Forest plot for clinical improvement at 6 months. M-H: Mantel-Haenszel test; Fixed: a fixed effects model, CI: confidence intervals.

failure that leads to the application of bail stenting especially in complex diseases [20]. Again, simple debulking therapy injures vascular endothelium and leads to smooth muscle cell proliferation, which caused a high recurrence rate. There is a complementarity between DCB and debulking therapy. The combination of DA or LD with subsequent DCB may have the advantage of removal occupation and antiproliferative effect. In 2013, Gandini et al. conducted a randomized controlled trial (RCT) comparing the efficacy of LD+DCB with
DCB, which is the only RCT to date [21]. The results indicated that $\mathrm{LD}+\mathrm{DCB}$ significantly increased the rate of patency $(6$ months: $91.7 \%$ vs $58.3 \%, P=0.01 ; 12$ months: $66.7 \%$ vs $37.5 \%, P=0.01)$ and decreased the rate of TLR (12 months: $16.7 \%$ vs $50 \%, P=0.01)$ compared with DCB alone. In 2013, Sixt et al. retrospectively evaluated the efficacy of DA+DCB with DA [22]. The results indicated that DA+DCB significantly increased the rate of patency at 12 months $(84.7 \%$ vs $43.8 \%, P<0.01)$ compared with DA alone. In 2017, 


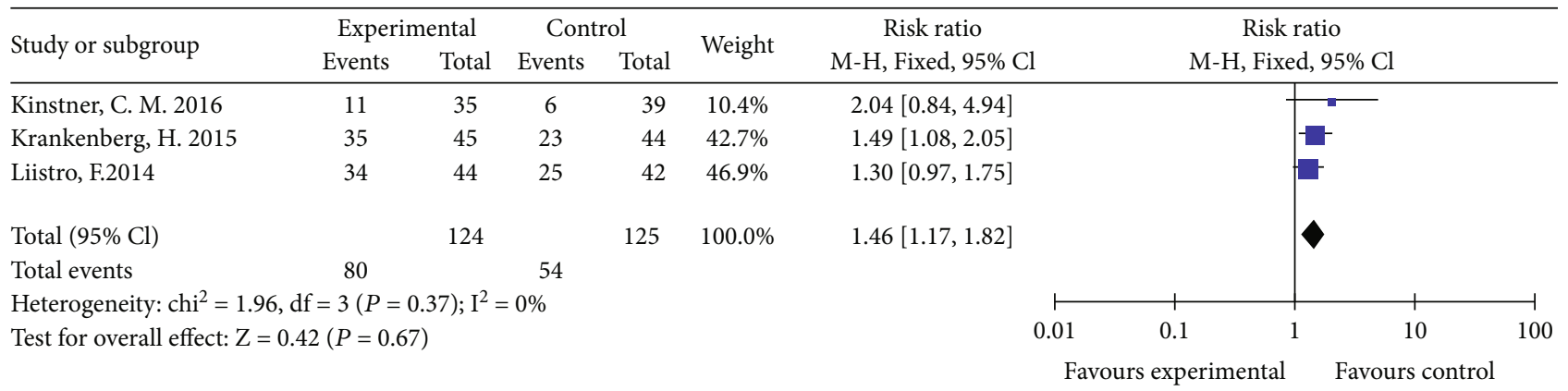

Figure 10: Forest plot for clinical improvement at 12 months. M-H: Mantel-Haenszel test; Fixed: a fixed effects model; CI: confidence intervals.

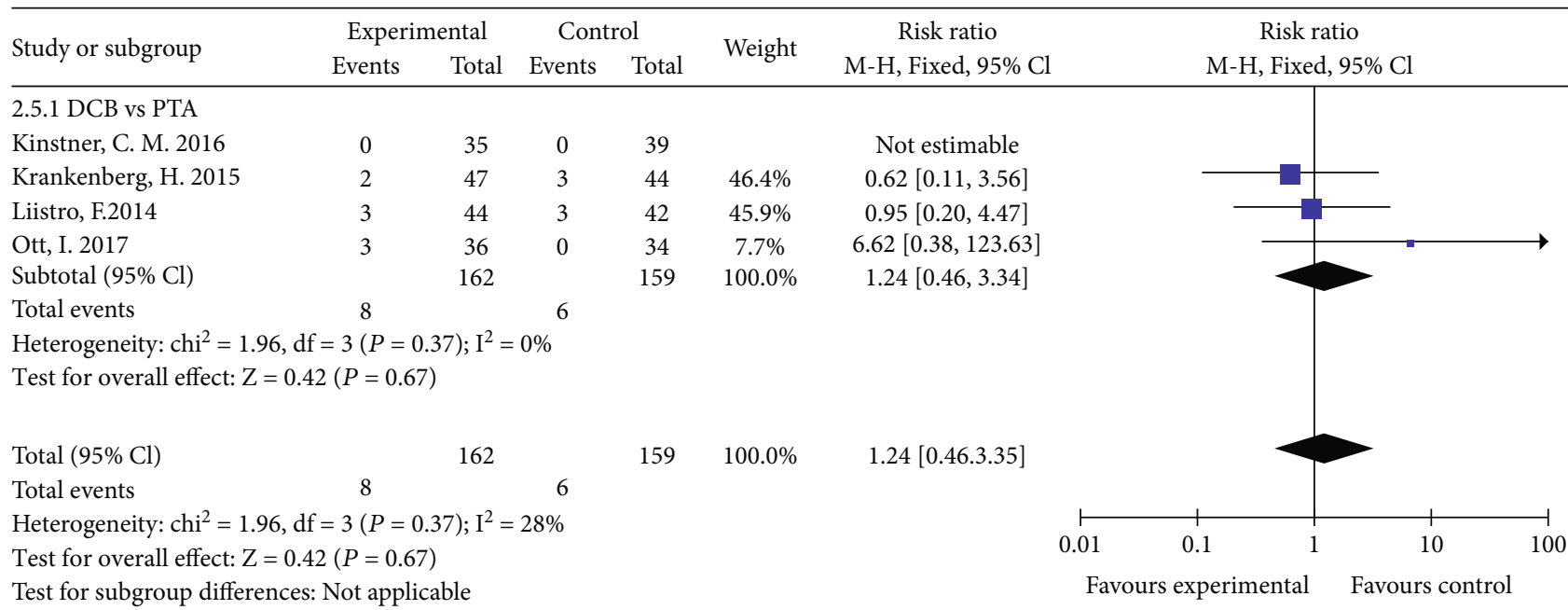

Figure 11: Forest plot for all-cause mortality. M-H: Mantel-Haenszel test; Fixed: a fixed effects model; CI: confidence intervals.

Kokkinidis, D. G. et al. retrospectively evaluated the efficacy of $\mathrm{LD}+\mathrm{DCB}$ with $\mathrm{LA}[23]$. The results indicated that $\mathrm{LD}+\mathrm{DCB}$ significantly increased the rate of patency $(86.7 \%$ vs $56.9 \%$, $P<0.01)$ and decreased the rate of TLR $(72.5 \%$ vs $50.5 \%$, $P<0.05)$ at 12 months compared with $\mathrm{LD}$ alone. These results all showed the possible superior of the combination of debulking with DCB. So a number of large-scale RCTs are needed to further evaluate this combined method.

Second, our meta-analysis showed the inconsistence of ABI outcome with other clinical and angiographic outcomes. This inconsistency may be caused by the lack of adequate trials and sample size and the fact that only two studies with 193 patients reported ABI data.

Third, the pooled results showed that DCB, LD, and PTA had a very low incidence of amputation and mortality, and no statistical difference was found between them, indicating DCB and LD are relatively safe methods. However, a study found $\mathrm{LD}+\mathrm{DCB}$ lowered the incidence of major amputation ( $8 \%$ vs $46 \%, P<0.01)$ and mortality $(12 \%$ vs $37 \%, P<0.05)$ compared with DCB alone in diabetic foot patients with critical limb ischemia. We found that the ISR in the study of Gandini et al. were all totally occluded, and the diseases were more complex and serious. So I think more trials are needed to evaluate the safety of LD+DCB, DCB alone, and debulking alone for patients with complex diseases and totally occluded ISR.
The present analysis has several limitations. First, there were small number of RCTs ( 5 trials with 599 participants) included in the meta-analysis. Second, Tosaka et al. classified FP-ISR into three types: class I: focal ISR ( $\leq 50 \mathrm{~mm}$ length), class II: diffuse ISR ( $\geq 50 \mathrm{~mm}$ in length), class III: totally occluded ISR [24]. The ISR Tosaka classification of patients in individual trials consisted of class I, class II, and class III. However, the trials did not conduct subgroup analysis according to the ISR Tosaka classification. Third, it is unclear whether the bailout stent was counted as TLR in some trials (PACUBA, DEBATE-ISR, and ISAR-PEBIS).

\section{Conclusion}

In conclusion, our meta-analysis showed that DCB and LD had superior clinical (freedom from TLR and clinical improvement) and angiographic outcomes (patency rate) compared with PTA for the treatment of femoropopliteal ISR. Moreover, DCB and LD had a low incidence of amputation and mortality and were relatively safe methods. However, considering their inherent shortcomings, the combination of DA or LD with DCB was attempted to be used and had an encouraging result. Thus, a number of large-scale RCTs are needed to further evaluate the efficiency and safety of this combined method. 


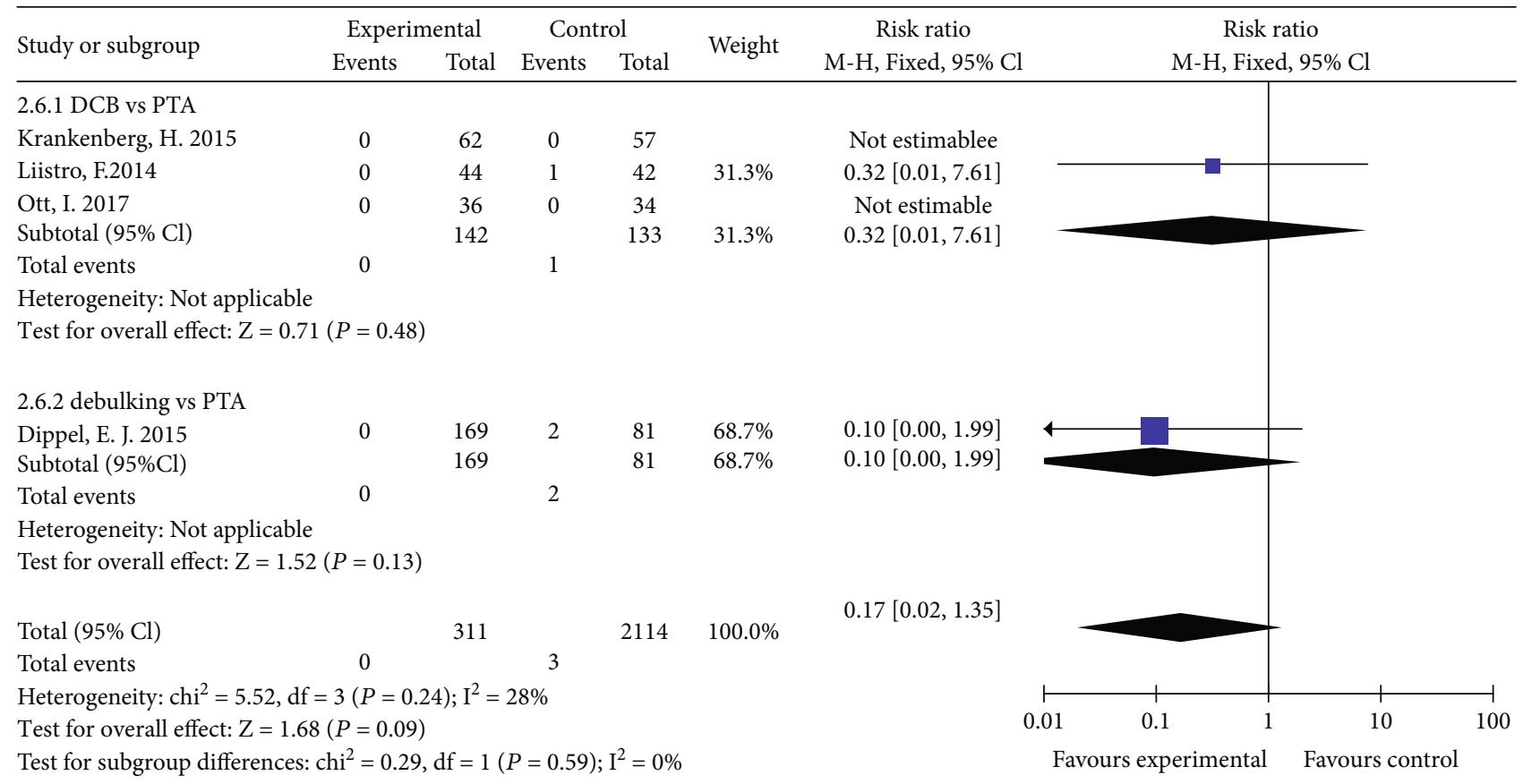

FIGURE 12: Forest plot for amputation. M-H: Mantel-Haenszel test; Fixed: a fixed effects model; CI: confidence intervals.

\section{Ethical Approval}

Ethics approval is not applicable. This study is a research on research study.

\section{Disclosure}

The author reports no conflicts of interest in this work.

\section{Conflicts of Interest}

The authors declare that they have no conflicts of interest.

\section{Supplementary Materials}

Figure S1: (funnel plot of patency at 6 months), Figure S2: (funnel plot of patency at 12 months), Figure S3: (funnel plot of freedom from TLR at 6 months), Figure S4: (funnel plot of freedom from TLR at 12 months), Figure S5: (funnel plot of clinical improvement at 6 months), Figure S6: (funnel plot of clinical improvement at 12 months), Figure S7: (funnel plot of ABI at 6 months), Figure S8: (funnel plot of ABI at 12 months), Figure S9: (funnel plot of all-cause mortality), Figure S10: (funnel plot of amputation), PRISMA flow diagram, and Table S1: (8 full-text excluded articles). (Supplementary Materials)

\section{References}

[1] M. K. Lichtenberg, J. G. Carr, and J. A. Golzar, "Optical coherence tomography: guided therapy of in-stent restenosis for peripheral arterial disease," The Journal of cardiovascular surgery, vol. 58, no. 4, pp. 518-527, 2017.
[2] M. Michael Lichtenberg, "Treating In-Stent Restenosis. Is laser atherectomy plus DCB the answer to achieving superior results in ISR treatment?," Supplement to Endovascular Today Europe, vol. 4, pp. 12-15.

[3] T. Zeller, M. D. Dake, G. Tepe et al., "Treatment of femoropopliteal in-stent restenosis with paclitaxel-eluting stents," Jacc Cardiovascular Interventions, vol. 6, no. 3, pp. 274-281, 2013.

[4] A. Rastan, J. F. McKinsey, L. A. Garcia et al., "One-year outcomes following directional atherectomy of infrapopliteal artery lesions: subgroup results of the prospective, multicenter DEFINITIVE LE trial," Journal of Endovascular Therapy, vol. 22, no. 6, pp. 839-846, 2015.

[5] S. Grotti, F. Liistro, P. Angioli et al., "Paclitaxel-eluting balloon vs standard angioplasty to reduce restenosis in diabetic patients with in-stent restenosis of the superficial femoral and proximal popliteal arteries: three-year results of the DEBATE-ISR study," Journal of Endovascular Therapy, vol. 23, no. 1, pp. 52-57, 2016.

[6] E. J. Armstrong, T. Thiruvoipati, K. Tanganyika, G. D. Singh, and J. R. Laird, "Laser atherectomy for treatment of femoropopliteal in-stent restenosis," Journal of Endovascular Therapy, vol. 22, no. 4, pp. 506-513, 2015.

[7] H. Krankenberg, T. Tübler, M. Ingwersen et al., "Drug-coated balloon versus standard balloon for superficial femoral artery in-stent restenosis: the randomized Femoral Artery In-Stent Restenosis (FAIR) trial," Circulation, vol. 132, no. 23, pp. 2230-2236, 2015.

[8] E. J. Dippel, P. Makam, R. Kovach et al., "Randomized controlled study of excimer laser atherectomy for treatment of femoropopliteal in-stent restenosis: initial results from the EXCITE ISR trial (EXCImer Laser Randomized Controlled Study for Treatment of FemoropopliTEal In-Stent Restenosis)," Jacc Cardiovascular Interventions, vol. 8, no. 1, pp. 92 101, 2015. 
[9] C. M. Kinstner, J. Lammer, A. Willfort-Ehringer et al., "Paclitaxel-eluting balloon versus standard balloon angioplasty in in-stent restenosis of the superficial femoral and proximal popliteal artery: 1-year results of the PACUBA trial," Jacc Cardiovascular Interventions, vol. 9, no. 13, pp. 1386-1392, 2016.

[10] J. Wetterslev, K. Thorlund, J. Brok, and C. Gluud, "Trial sequential analysis may establish when firm evidence is reached in cumulative meta-analysis," Journal Of Clinical Epidemiology, vol. 61, no. 1, pp. 64-75, 2008.

[11] F. Liistro, P. Angioli, I. Porto et al., "Paclitaxel-eluting balloon vs. standard angioplasty to reduce recurrent restenosis in diabetic patients with in-stent restenosis of the superficial femoral and proximal popliteal arteries: the DEBATE-ISR study," Journal of Endovascular Therapy, vol. 21, no. 1, pp. 1-8, 2014.

[12] I. Ott, S. Cassese, P. Groha et al., "ISAR-PEBIS (paclitaxel-eluting balloon versus conventional balloon angioplasty for instent restenosis of superficial femoral artery): a randomized trial," Journal of the American Heart Association, vol. 6, no. 7, 2017.

[13] G. D. Singh, E. J. Armstrong, and J. R. Laird, "Femoropopliteal in-stent restenosis: current treatment strategies," The Journal of Cardiovascular Surgery, vol. 55, no. 3, pp. 325-333, 2014.

[14] R. A. Byrne, F. J. Neumann, J. Mehilli et al., "Paclitaxel-eluting balloons, paclitaxel-eluting stents, and balloon angioplasty in patients with restenosis after implantation of a drug-eluting stent (ISAR-DESIRE 3): a randomised, open-label trial," The Lancet, vol. 381, no. 9865, pp. 461-467, 2013.

[15] B. Scheller, Y. P. Clever, B. Kelsch et al., "Long-term follow-up after treatment of coronary in-stent restenosis with a paclitaxel-coated balloon catheter," Jacc Cardiovascular Interventions, vol. 5, no. 3, pp. 323-330, 2012.

[16] N. I. Akkus, A. Abdulbaki, E. Jimenez, and N. Tandon, "Atherectomy devices: technology update," Medical Devices, vol. 8, pp. 1-10, 2015.

[17] R. M. Dave, R. Patlola, K. Kollmeyer et al., "Excimer laser recanalization of femoropopliteal lesions and 1-year patency: results of the CELLO registry," Journal of Endovascular Therapy, vol. 16, no. 6, pp. 665-675, 2009.

[18] S. Hajibandeh, S. Hajibandeh, S. A. Antoniou, F. Torella, and G. A. Antoniou, "Treatment strategies for in-stent restenosis in peripheral arterial disease: a systematic review," Interactive Cardiovascular and Thoracic Surgery, vol. 28, no. 2, pp. 253261, 2019.

[19] C. Nicolais, V. Lakhter, H. U. H. Virk et al., "Therapeutic options for in-stent restenosis," Current Cardiology Reports, vol. 20, no. 2, 2018.

[20] R. Dattilo, S. I. Himmelstein, and R. F. Cuff, "The COMPLIANCE $360^{\circ}$ Trial: a randomized, prospective, multicenter, pilot study comparing acute and long-term results of orbital atherectomy to balloon angioplasty for calcified femoropopliteal disease," The Journal of Invasive Cardiology, vol. 26, no. 8, pp. 355-360, 2014.

[21] R. Gandini, C. Del Giudice, S. Merolla, D. Morosetti, E. Pampana, and G. Simonetti, "Treatment of chronic SFA in-stent occlusion with combined laser atherectomy and drug-eluting balloon angioplasty in patients with critical limb ischemia: a single-center, prospective, randomized study," Journal of Endovascular Therapy, vol. 20, no. 6, pp. 805-814, 2013.

[22] S. Sixt, O. G. Carpio Cancino, A. Treszl et al., "Drug-coated balloon angioplasty after directional atherectomy improves outcome in restenotic femoropopliteal arteries," Journal of Vascular Surgery, vol. 58, no. 3, pp. 682-686, 2013.

[23] D. G. Kokkinidis, P. Hossain, O. Jawaid et al., "Laser atherectomy combined with drug-coated balloon angioplasty is associated with improved 1-year outcomes for treatment of femoropopliteal in-stent restenosis," Journal of Endovascular Therapy, vol. 25, no. 1, pp. 81-88, 2017.

[24] A. Tosaka, Y. Soga, O. Iida et al., "Classification and clinical impact of restenosis after femoropopliteal stenting," Journal of the American College of Cardiology, vol. 59, no. 1, pp. 1623, 2012. 